\title{
The glycerophosphoinositols: from lipid metabolites to modulators ofT-cell signaling
}

\section{Laura Patrussi $^{1}{ }^{*}$, Stefania Mariggiò $^{2}{ }^{*}$, Daniela Corda $^{2}$ and Cosima T. Baldari ${ }^{1}$}

1 Department of Life Sciences, University of Siena, Siena, Italy

2 Institute of Protein Biochemistry, National Research Council, Naples, Italy

\section{Edited by:}

Klaus Okkenhaug, Babraham

Institute, UK

\section{Reviewed by:}

Sho Yamasaki, Kyushu University, Japan

Karsten Sauer, The Scripps Research Institute, USA

\section{*Correspondence:}

Laura Patrussi, Department of Life

Sciences, University of Siena, Via

Aldo Moro 2, 53100 Siena, Italy

e-mail:patrussi2@unisi.it;

Stefania Mariggiò, Institute of Protein

Biochemistry, National Research

Council, Via Pietro Castellino 111,

80131 Naples, Italy

e-mail:s.mariggio@ibp.cnr.it
Glycerophosphoinositols (GPIs) are bioactive, diffusible phosphoinositide metabolites of phospholipase $A_{2}$ that act both intracellularly and in a paracrine fashion following their uptake by specific transporters. The most representative compound, glycerophosphoinositol (GroPlns), is a ubiquitous component of eukaryotic cells that participates in central processes, including cell proliferation and survival. Moreover, glycerophosphoinositol 4phosphate (GroPIns4P) controls actin dynamics in several cell systems by regulating Rho GTPases. Recently, immune cells have emerged as targets of the biological activities of the GPIs. We have shown that exogenous GroPIns4P enhances CXCL12-induced T-cell chemotaxis through activation of the kinase Lck in a cAMP/PKA-dependent manner. While highlighting the potential of GroPIns $4 P$ as an immunomodulator, this finding raises questions on the role of endogenously produced GroPIns4P as well as of other GPIs in the regulation of the adaptive immune responses under homeostatic and pathological settings. Here we will summarize our current understanding of the biological activities of the GPIs, with a focus on lymphocytes, highlighting open questions and potential developments in this promising new area.

\section{Keywords: glycerophosphoinositol, T-cell chemotaxis, CXCL12, Lck}

The glycerophosphoinositols (GPIs) are ubiquitous water-soluble phosphoinositide metabolites produced by all eukaryotic cells (1-3). Not surprisingly considering their central role in the orchestration of signaling cascades, among the phosphoinositides it is the inositol phosphates that have monopolized the scene. Accumulating evidence has however highlighted a role for the GPIs as modulators of important biological functions in a number of cell types, including T-lymphocytes, in both physiological and pathological settings. Here we will summarize our current understanding of the metabolic pathways that regulate GPI production and discuss their biological activities, focusing on T-cells.

\section{BIOSYNTHESIS, TRANSPORT AND DEGRADATION OF GPIS}

The GPIs, which include glycerophosphoinositol (GroPIns) and its phosphorylated derivatives glycerophosphoinositol 4-phosphate (GroPIns4P) and glycerophosphoinositol 4,5bisphosphate (GroPIns4,5P2), are generated from membrane phosphoinositides through two sequential deacylation reactions that are carried out by a phospholipase $\mathrm{A}_{2}\left(\mathrm{PLA}_{2}\right)$ and a lysophospholipase (2). Studies on the most abundant of these metabolites, GroPIns, have provided evidence that both of these reactions can be catalyzed by the same enzyme, which has been identified in thyroid cells and macrophages as the $\alpha$ isoform of group IV PLA 2 $\left(\mathrm{PLA}_{2} \mathrm{IV} \alpha\right)[(4,5)$; Figure 1]. We have recently shown that the same enzymatic pathway is responsible for GroPIns4P production in macrophages upon treatment with a pro-inflammatory stimulus (unpublished results; Figure 1).

Glycerophosphoinositols can interact with intracellular targets and/or be released into the extracellular medium through specific membrane transporters, following their chemical gradient. The GroPIns transporter, which is responsible for the bidirectional transfer of GroPIns, was initially identified in yeast (6), and its human ortholog is the Glut2 permease (7). Reasonably, Glut2 represents only one of the mammalian GroPIns transporters, as it has cell-specific expression patterns, while GroPIns membrane permeation appears to be a general process. There is also evidence of GroPIns $4 P$ membrane transport. Although no specific transporter for GroPIns4P has been identified to date, several GroPIns $4 P$-mediated activities show biochemical features that indicate specific, transporter-mediated mechanisms (8).

The half-life of GPIs is relatively short both inside the cell and in the extracellular milieu. This applies in particular to the phosphorylated, biologically active derivative, GroPIns $4 P$ which is rapidly metabolized within the cell, undergoing dephosphorylation to GroPIns through a $\mathrm{Ca}^{2+}$-dependent and GroPIns4P-selective activity associated with the cell membrane fraction (Figure 1). Alternatively, a $\mathrm{Ca}^{2+}$-insensitive activity leads to the phosphorylation of GroPIns $4 P$ to GroPIns4,5 $P_{2}$ (8). GroPIns can be reacylated to phosphatidylinositol (PtdIns) both in whole cells and in membrane fractions. At variance, no detectable reacylation of GroPIns $4 P$ has been documented to date (8).

The glycerophosphodiesterases GDE1 and GDE3, both of which are membrane-bound ectoenzymes, catalyze the hydrolysis of extracellular GroPIns (9, 10). GDE1 is ubiquitously expressed and hydrolyzes GroPIns to produce inositol and glycerol phosphate (9). GDE1 activity is regulated by G-protein-coupled receptors, and it is stimulated by $\beta$-adrenergic receptor agonists but inhibited by $\alpha$-adrenergic receptor agonists and lysophosphatidic 


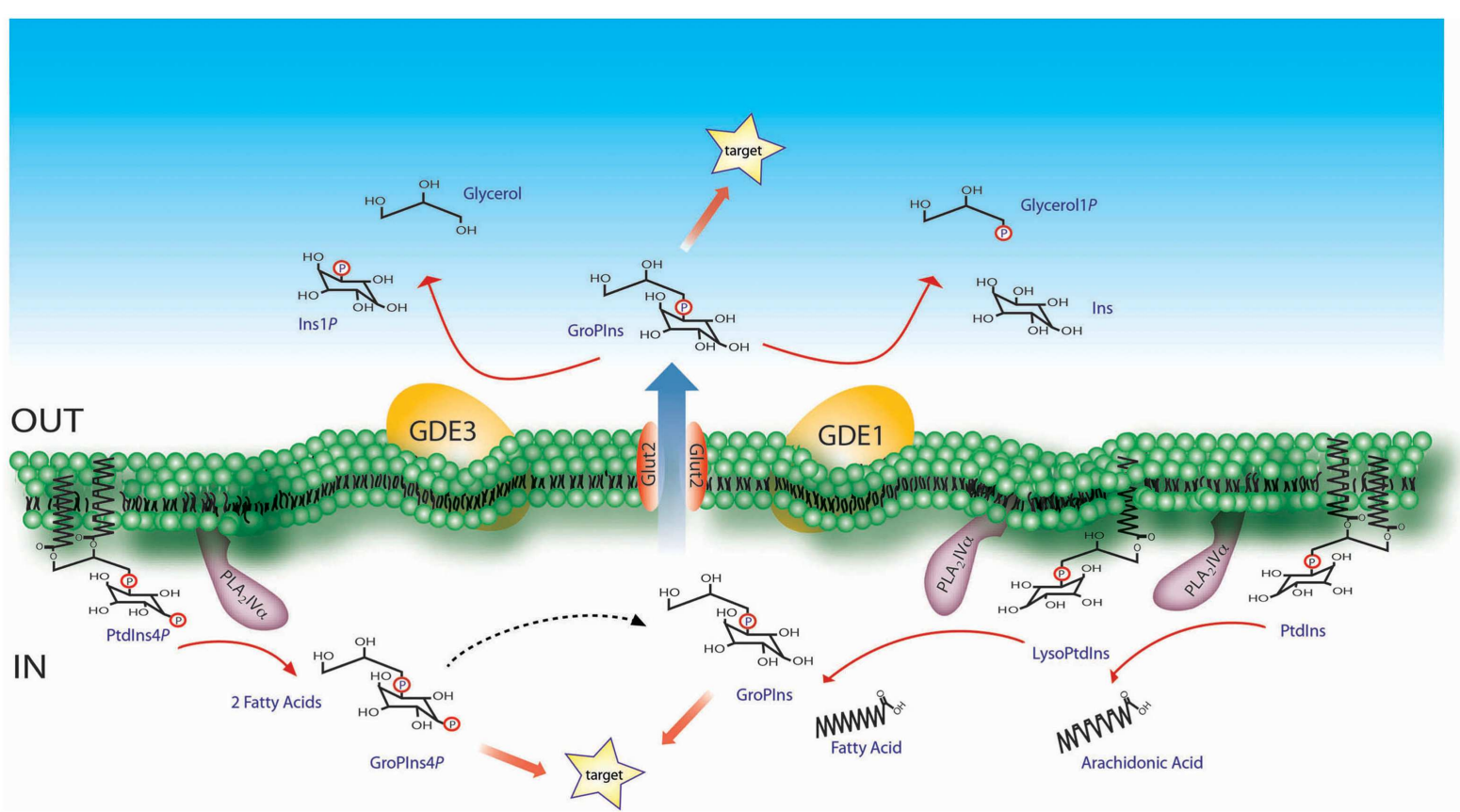

FIGURE 1 | Schematic representation of the GroPIns metabolism. The formation of GroPIns occurs from membrane phosphatidylinositol (Ptdlns) via two sequential steps, both of which are catalyzed by $P L A_{2} I V \alpha$. The first deacylation produces lysophosphatidylinositol (LysoPtdlns) and free arachidonic acid, since PLA $\mathrm{IV}_{2} \alpha$ selectively hydrolyzes phosphoinositides substituted in the sn-2 position with arachidonic acid (49). The second deacylation releases free fatty acid and GroPIns. As indicated, $P L A_{2} I V \alpha$ supports both of these deacylation steps, as demonstrated in in vitro investigations using purified phosphoinositide and lysophosphatidylinositol substrates together with the recombinant enzyme (4). Once produced in the cytoplasm, GroPIns can be active on intracellular targets or can be released through the Glut2 transporter into the extracellular space, where it can act as a paracrine factor on nearby target cells. The subsequent catabolism of GroPIns is instead located on the extracellular side of the plasma-membrane and is mediated by the GDEs. GroPIns4P formation, also schematized, occurs starting from membrane phosphatidylinositol 4-phosphate which is hydrolyzed, as for GroPIns, by PLA $\mathrm{IV}_{2}$ (unpublished observations, see main text for details). acid, thus providing a further level of modulation of GroPIns metabolism (9). GDE3 is a marker of osteoblast differentiation, and is predominantly expressed in mature osteocytes (11). GDE3 hydrolyzes GroPIns with a different type of attack of the phosphodiester bond, which produces inositol phosphate and glycerol (10). GroPIns $4 P$ is not substrate of GDE1 or GDE3, but it can compete with GroPIns for its hydrolysis by these glycerophosphodiesterases $(9,10)$.

\section{GPI PRODUCTION IN IMMUNE CELLS GroPIns IS PRODUCED BY MACROPHAGES IN RESPONSE TO PRO-INFLAMMATORY STIMULI}

A number of pharmacological and pro-inflammatory stimuli have been shown to trigger phosphoinositide hydrolysis in macrophages (2). Similar to other cell types, GroPIns production in these cells is regulated by a $\mathrm{Ca}^{2+}$-dependent pathway involving the $\mathrm{PLA}_{2}$-catalyzed deacylation of PtdIns (2). Studies on macrophages treated with cholera or pertussis toxin provided evidence that $\mathrm{PLA}_{2}$ is activated downstream of $\mathrm{G}$ proteins, catalyzing the hydrolysis of PtdIns and leading to the production of arachidonic acid derivatives and GroPIns (12). A similar pathway was identified in Kupffer cells, the resident macrophages of the liver, following stimulation with inflammatory mediators produced upon bacterial endotoxin challenge $(13,14)$. A concerted activation of the arachidonate pathway and production of GroPIns has been reported in several other cell types (2).

$\mathrm{PLA}_{2} \mathrm{IV} \alpha$, which had been identified as the specific, $\mathrm{Ca}^{2+}$ dependent $\mathrm{PLA}_{2}$ responsible for GroPIns production in thyroid cells (4), has been recently demonstrated to carry out this function also in macrophages. Zizza and colleagues (5) showed that $\mathrm{PLA}_{2} \mathrm{IV} \alpha$, which is abundantly expressed in macrophages, is phosphorylated by the MAP kinases Erk1/2 and by the stress-activated kinases $\mathrm{p} 38$ and JNK and translocates to the membrane of nascent phagosomes during Fc-Receptor (FcR)-mediated phagocytosis. A selective PLA $\mathrm{P}_{2} \mathrm{IV} \alpha$ activation was observed to also occur upon LPS treatment which, similar to FcR engagement, triggers arachidonic acid release (5). Moreover, pharmacological inhibition of PLA 2 IV $\alpha$ completely abolished both LPS- and phagocytosismediated GroPIns production. Interestingly, a time course analysis of GroPIns production during FcR-mediated phagocytosis revealed a persistent increase in the levels of intracellular GroPIns over time, which was paralleled by GroPIns release into the extracellular medium (our unpublished observations). This suggests that GroPIns may participate in the inflammatory responses of macrophages by acting not only in an autocrine manner, but also as a paracrine factor.

The intracellular levels of the GPIs have also been measured in T-cells. Mass spectrometry data showed that Jurkat 
T-cells are among the cell lines with low intracellular levels of GroPIns $(45 \pm 1 \mu \mathrm{M})(15,16)$. Moreover, these basal levels are not increased by known pharmacological activators of $\mathrm{PLA}_{2} \mathrm{IV} \alpha$, such as $\mathrm{Ca}^{2+}$ ionophores, or by chemotactic stimuli, such as CXCL12, which suggests that a $\mathrm{Ca}^{2+}$-independent enzyme is involved in GroPIns production in these cells. Alternatively, the concentrations of arachidonoyl-substituted PtdIns, the GPI precursor, may not be sufficient to produce significant increases in the levels of intracellular GroPIns.

\section{MODULATION OF GroPIns PRODUCTION DURING IMMUNE CELL DIFFERENTIATION}

Phospholipase $\mathrm{A}_{2}$ activation is not only triggered by plasmamembrane receptors but also occurs during cell differentiation (17-19). Mountford and colleagues provided evidence that the levels of phosphoinositides change during the differentiation of both myeloid and lymphoid cells (20-22). Using HL60 promyelocytic cells, which can differentiate either to neutrophils in response to all-trans retinoic acid and granulocyte-colony-stimulating factor or to monocytes in response to $1 \alpha-25$-dihydroxyvitamin $\mathrm{D}_{3}$, they showed that the intracellular GroPIns levels increased in the early stages of differentiation to either lineages, eventually doubling in fully differentiated cells. Consistent with these findings, GroPIns levels increased in neutrophils that spontaneously differentiated in culture, as compared to the initial blasts (22). Similar experiments, carried out on paired cell lines representative of immature and mature states of B-lymphocytes (Ba/F3 and NSI cells) and T-lymphocytes (S49 and C8166 cells) showed substantial increases in GroPIns levels in the cells representative of the mature states (22). Although more accurate methods to quantitate GroPIns as well as more physiological differentiation conditions will be required to validate these data, the changes in the concentrations of intracellular GroPIns suggest a role for this metabolite in the regulation of both myeloid and lymphoid cell differentiation.

\section{GroPIns4P PRODUCTION BY MACROPHAGES}

In addition to GroPIns, its monophosphorylated derivative, GroPIns $4 P$, has been detected in several cell types, including macrophages [(2); our unpublished observations)]. $\operatorname{PLA}_{2} \operatorname{IV} \alpha$, is responsible for the production of both GPIs (i.e., GroPIns and GroPIns4P), based on the relative availability of the respective lipid precursors (PtdIns and PtdIns4P, respectively) [(4, 5); our unpublished observations]. A conundrum in these studies are the technical limitations in the accurate measurement of these metabolites. For example, the relatively high levels of GroPIns make the detection of small increases more difficult, which might explain why increases in GroPIns $4 P$ (which generally represents $<3 \%$ of the total GPIs) do not always appear to be paralleled by increases in GroPIns. The rapid metabolism of GroPIns $4 P$ is a further drawback for precise determinations of its levels, although this was partially overcome by performing GroPIns $4 P$ measurements in the presence of orthovanadate, a general phosphatase inhibitor (2), which made it possible to monitor GroPIns4P increases in macrophages exposed to LPS (our unpublished observation). At variance with macrophages, no detectable production of GroPIns4P can be observed in T-cells (16).

\section{THE GPIS AS MODULATORS OF T-CELL FUNCTIONS: FACTS} AND HYPOTHESES

\section{GroPInS4P PROMOTES ACTIN POLYMERIZATION IN T-LYMPHOCYTES}

Cortical actin rearrangements, which are regulated by the Rho family of small GTPases (23), are crucial for a number of processes that orchestrate T-lymphocyte activation and motility $(24,25)$. Exogenous administration of GroPIns $4 P$ to fibroblasts induces the formation of actin ruffles and stress fibers by modulating the activity of Rac and Rho $(26,27)$, suggesting a potential role for this phosphoinositide derivative in the regulation of the actin cytoskeleton in other cell types. Treatment of both Jurkat Tcells and peripheral blood lymphocytes from healthy donors with GroPIns $4 P$ induces indeed actin polymerization (16), suggesting that the processes involving F-actin dynamics, including redistribution of components associated with lymphocyte motility and immune synapse assembly might be modulated by GroPIns4P.

The ability of GroPIns4P to promote actin polymerization in T-cells stems, at least in part, from its ability to induce the phosphorylation of the GDP/GTP exchanger Vav (16), which controls the activation of Rac and Cdc42 in hematopoietic cells (28). These data are consistent with the finding that GroPIns $4 P$ triggers a signaling cascade in fibroblasts that leads to plasma-membrane translocation of Tiam1, a Rac-specific GDP/GTP exchanger in these cells (27). This activity provides a mechanistic explanation of the agonistic effects of GroPIns $4 P$ on the actin cytoskeleton dynamics.

The ability of GroPIns4P to promote Vav activation suggests the possibility that it may also modulate gene expression. Vav initiates indeed a pathway involving recruitment to active Rac of the serine/threonine kinase Pak1, which triggers the activation of p38 and JNK that directly or indirectly activate a number of transcription factors (29). Consistent with this notion, T-cell treatment with GroPIns $4 P$ resulted in activation of both p38 and JNK, with a similar time course as Vav (16).

\section{VAV ACTIVATION BY GroPInS4P REQUIRES LCK}

Tyrosine phosphorylation of Vav is mediated by the cooperative activity of Syk and Src family protein tyrosine kinases (PTKs) (28). We have shown that treatment of T-cells with GroPIns4P results in enhanced Lck activity [(16); Figure 2]. Although other mechanisms may account for Vav activation, this event is likely to be causal to the agonistic activity of GroPIns4P on Vav activation and the resulting actin cytoskeleton rearrangements. GroPIns $4 P$ fails indeed to trigger Vav phosphorylation in the Lck-deficient Jurkat T-cell variant JCaM.1 (16, 30). Moreover, GroPIns4P triggers Src phosphorylation in fibroblasts, which is required for plasma-membrane translocation of Tiam1 (27). Lck activation, as well as Src activation, is not a direct effect of GroPIns $4 P$, at least as assessed in vitro (16). Unfortunately, while these studies have restricted the field, no final mechanism of action for the GPIs can be postulated, until a proteomic approach to identify direct interactors can be completed.

\section{GroPIns4P TARGETS LCK-DEPENDENT SIGNALING BY MODULATING cAMP}

In quiescent T-cells Lck is kept in an inactive state by the inhibitory kinase Csk, which becomes phosphorylated and activated by the cAMP-dependent serine/threonine protein kinase A (PKA) (31). 


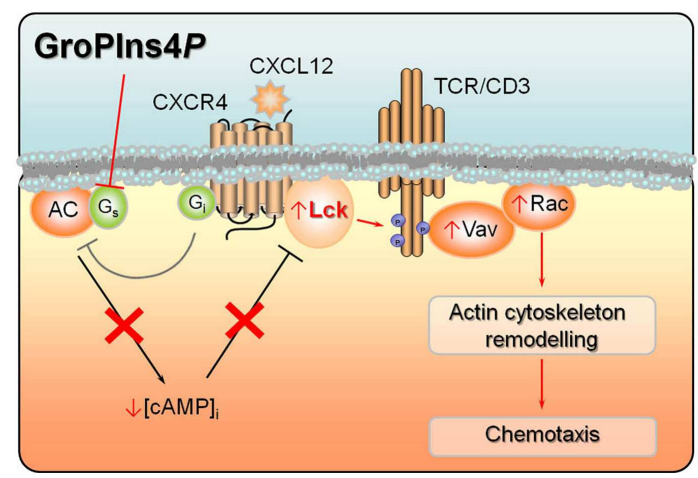

FIGURE 2 | Convergence of signals by CXCL12 and GroPIns4P on adenylate cyclase activity. CXCL12 binding to its cognate receptor CXCR4 leads to activation of Lck, which stably interacts with the receptor. Lck in turn phosphorylates multiple tyrosine residues in the cytosolic tails of the TCR/CD3 complex, thereby triggering a signaling cascade involving Vav activation and eventually actin cytoskeletal rearrangements. Lck activity is further potentiated by CXCR4-dependent stimulation of Gi protein which, by inhibiting adenylate cyclase, lowers the levels of intracellular CAMP, resulting in decreased PKA-dependent activation of Csk, a negative regulator of Lck. GroPIns4P potentiates migratory signaling by CXCL12 by blocking the activity of $\mathrm{Gs}$ protein, thereby further lowering adenylate cyclase activity and hence the intracellular CAMP levels and contributing to Lck activation. Phosphorylation states and events are shown as small blue circles.

Activation events are shown as arrows, inhibition events as truncated lines.

We showed that treatment of Jurkat T-cells with GroPIns4P results in a decrease in the levels of cAMP, leading to impaired PKA activation and Csk phosphorylation. Hence the agonistic activity of GroPIns $4 P$ on Lck activation and downstream signaling results from its ability to inhibit cAMP production (16). These results are consistent with the finding that GroPIns $4 P$ (but not GroPIns or GroPIns $\left.4,5 P_{2}\right)$ inhibits adenylate cyclase activity in other cell types $(32,33)$. It is noteworthy that cAMP/PKA-mediated modulation of molecules downstream of Lck may also contribute to its effects on F-actin dynamics in T-cells (34), including Rho itself, which is phosphorylated by PKA on an inhibitory residue $(35,36)$.

The ability of GroPIns4P to activate Lck, which is responsible for initiation of the T-cell receptor (TCR) signaling cascade, strongly suggests that other signaling events, in addition to the Vav/p38/JNK pathway, might be triggered by GroPIns4P. One of the key targets of Lck both in TCR (37) and chemokine receptor signaling (38) is the PTK ZAP-70, which couples these receptors to multiple signaling pathways, including the Ras/MAP kinase pathway (39). GroPIns4P induces indeed the activation of ZAP70 and the adaptor Shc (16), which interacts with, and becomes phosphorylated by ZAP-70 in response to TCR engagement and contributes to Ras activation by recruiting the Grb2/Sos complex (40). Consistent with its ability to promote Shc phosphorylation, GroPIns4P activates Erk1/2. These effects of GroPIns4P are crucially dependent on Lck, as they fail to occur in JCaM.1 cells (16).

\section{GroPIns4P ENHANCES CXCR4 SIGNALING}

The ability of GroPIns $4 P$ to promote actin polymerization in T-cells profoundly influences their responses to chemokines.
GroPIns4P (but not the other GPIs) enhances indeed CXCR4dependent chemotaxis toward CXCL12 (16), a chemokine that regulates lymphocyte homing to secondary lymphoid organs under homeostatic conditions (39). Interestingly, the two signals may converge, at least in part, on adenylate cyclase. In fact, CXCL12-mediated activation of CXCR4 promotes the release of the $\alpha$-subunit of heterotrimeric inhibitory $G$ protein $\left(\mathrm{G}_{\mathrm{i}}\right)$, thereby inhibiting adenylate cyclase activity (39). Moreover, in Jurkat Tcells GroPIns4P reverses the cAMP-elevating activity of cholera toxin, which ADP-ribosylates the $\alpha$-subunit of stimulatory $G$ proteins $\left(G_{s}\right)$ resulting in their persistent activation. This indicates that GroPIns4P is able to decrease cAMP production and cAMP/PKA-dependent Csk activation by inhibiting $\mathrm{G}_{s}$. These mechanisms can account for the additive effect of CXCL12 and GroPIns4P on Lck activation [(16); Figure 2].

Recent evidence supports a crosstalk between the TCR and CXCR4. The TCR is indeed transactivated by CXCR4 which then uses the TCR machinery to elicit and potentiate downstream signaling $(38,41)$. Similar to other G-protein-coupled receptors, CXCR4 directly interacts with and activates Lck. The association of CXCR4 with the TCR at the cell surface allows Lck to localize in close contact with the intracellular domains of the TCR/CD3 complex and phosphorylate $\mathrm{CD} 3 \zeta$, thereby triggering downstream signaling (39). By promoting Lck activation GroPIns4P might potentiate the ability of CXCR4 to transactivate the TCR and hence enhance the signaling cascades leading to T-cell chemotaxis, including the pathway leading to the Lck-dependent recruitment of Shc to the CXCR4/TCR dimer.

\section{GroPIns4P PRODUCTION BY BYSTANDER CELLS: A POTENTIAL MECHANISM TO CONTROL T-CELL RESPONSES IN THE LOCAL MICROENVIRONMENT}

How can the evidence obtained using exogenously added GroPIns $4 P$ be related to the physiological context of T-cell trafficking? Measurements of the GPI levels in Jurkat T-cells using a quantitative mass spectrometry approach revealed that these cells are among those with the lowest intracellular levels of GroPIns (15). At variance with T-cells, macrophages produce large amounts of GPIs in response to pro-inflammatory stimuli $(2,5)$, generating a gradient for their transporter-mediated release into the extracellular medium $(2,15,42)$. We propose that these macrophage-derived GPIs may act as paracrine factors for lymphocytes. In this scenario, GPIs produced at the site of infection would enhance effector T-cell recruitment, and thereby contribute to bacterial clearance. Interestingly, among the highest GPI producers are certain tumor cells $(2,43)$. The release of these metabolites, combined with chemotactic signals provided by the tumor microenvironment (44), could be hypothesized to promote T-cell infiltration and activation of anti-tumor immunity.

\section{GPIS AND T-CELL FATE: A WORKING HYPOTHESIS}

By promoting the activation of Lck, exogenous administration of GroPIns4P to T-lymphocytes triggers a Rho-family dependent pathway that is integrated with chemokine receptor signaling to potentiate T-cell chemotaxis (16). Given the central role of Lck as the initiator kinase in TCR signaling (37), it can be hypothesized that GroPIns4P has the ability to modulate T-cell activation. 
Lck has also been implicated in the regulation of T-cell apoptosis induced by a wide range of stimuli, including prolonged TCR stimulation (45) and treatment with sphingosine (46), and it is also an essential component of the signaling pathways that control $\mathrm{Ca}^{2+}$ mediated T-cell apoptosis, which involve both the conformational activation of Bax and the expression of proapoptotic Bcl-2 family members (47). How TCR engagement can lead to cell fates as diverse as activation, anergy, and apoptosis is one of the fundamental and as yet open questions in immunology. Investigating the effects of GroPIns4P, as well as of the other GPIs, on these processes, may provide valuable information on how TCR signaling is fine-tuned by these phosphoinositide metabolites to elicit different biological outcomes.

\section{CONCLUSION}

The interesting scenarios opened by the studies outlined in this review using exogenously added GPIs underscore the need to address the physiological function of endogenous GPIs in the modulation of immune cell function. It has however to be underlined that the results summarized in the present review, while in part validated on normal peripheral T-cells, have been largely obtained by exogenous administration of GroPIns4P to Jurkat Tcells, which are known to be defective in the activity of enzymes

\section{REFERENCES}

1. Berrie CP, Dragani LK, van der Kaay J, Iurisci C, Brancaccio A, Rotilio D, et al., Maintenance of PtdIns45P2 pools under limiting inositol conditions, as assessed by liquid chromatographytandem mass spectrometry and PtdIns45P2 mass evaluation in Ras-transformed cells. Eur J Cancer (2002) 38:2463-75. doi:10.1016/ S0959-8049(02)00485-9

2. Corda D, Iurisci C, Berrie CP. Biological activities and metabolism of the lysophosphoinositides and glycerophosphoinositols. Biochim Biophys Acta (2002) 1582:52-69. doi: 10.1016/S1388-1981(02)00137-3

3. Dragani LK, Berrie CP, Corda D, Rotilio D. Analysis of glycerophosphoinositol by liquid chromatography-electrospray ionisation tandem mass spectrometry using a beta-cyclodextrinbonded column. I Chromatogr B Analyt Technol Biomed Life Sci (2004) 802:283-9. doi: 10.1016/j.jchromb.2003.12.002

4. Mariggio S, Sebastia J, Filippi BM, Iurisci C, Volonte C, Amadio S, et al., A novel pathway of cell growth regulation mediated by a PLA2alphaderived phosphoinositide metabolite. FASEB J (2006) 20:2567-9. doi: 10.1096/fj.05-5397fje

5. Zizza P, Iurisci C, Bonazzi M, Cossart P, Leslie CC, Corda D, et al., Phospholipase A2IValpha regulates phagocytosis independent of its enzymatic activity. J Biol Chem
(2012) 287:16849-59. doi:10.1074/ jbc.M111.309419

6. Patton-Vogt JL, Henry SA. GIT1, a gene encoding a novel transporter for glycerophosphoinositol in Saccharomyces cerevisiae. Genetics (1998) 149:1707-15.

7. Mariggio S, Iurisci C, Sebastia J, Patton-Vogt J, Corda D. Molecular characterization of a glycerophosphoinositol transporter in mammalian cells. FEBS Lett (2006) 580:6789-96. doi:10.1016/j.febslet. 2006.11.039

8. Berrie CP, Iurisci C, Corda D. Membrane transport and in vitro metabolism of the Ras cascade messenger, glycerophosphoinositol 4-phosphate. Eur J Biochem (1999) 266:413-9. doi: 10.1046/j.1432-1327.1999.00870.x

9. Zheng B, Berrie CP, Corda D, Farquhar MG. GDE1/MIR16 is a glycerophosphoinositol phosphodiesterase regulated by stimulation of $\mathrm{G}$ protein-coupled receptors. Proc Natl Acad Sci U S A (2003) 100:1745-50. doi:10.1073/ pnas.0337605100

10. Corda D, Kudo T, Zizza P, Iurisci C, Kawai E, Kato N, et al., The developmentally regulated osteoblast phosphodiesterase GDE3 is glycerophosphoinositol-specific and modulates cell growth. J Biol Chem (2009) 284:24848-56. doi: 10.1074/jbc.M109.035444

11. Yanaka N. Mammalian glycerophosphodiester phosphodiesterases. Biosci Biotechnol

critically involved in lipid signaling, such as PTEN (48). We have therefore to take into account a possible impact of this defect on global lipid signaling, which might also involve GPI metabolism. Since, among immune cells, the major producers of GPIs are macrophages, it will be interesting to assess the impact of this physiological source of exogenous GPIs on normal T-cells in co-culture experiments. Unfortunately the study of immune modulation by GPIs in vivo, which in principle could be approached using $\mathrm{PLA}_{2} \mathrm{IV} \alpha^{-1-}$ mice, is prevented by lack of effect of PLA $\mathrm{PV}_{2} \alpha$ deficiency on GPI synthesis, at least in this model. This finding is likely to be accounted for by the fact that in $\mathrm{PLA}_{2} \mathrm{IV}^{-l-}$ cells GPI synthesis is taken over by the calcium-independent $\mathrm{PLA}_{2} \mathrm{VI}$, suggesting that a compensatory mechanism is activated under these conditions (15). A way to circumvent this problem in the future could be to generate an inducible knockout mouse lacking $\mathrm{PLA}_{2} \mathrm{IV} \alpha$ expression in macrophages and/or T-cells, or alternatively to modulate the expression of the specific GDEs.

\section{ACKNOWLEDGMENTS}

Part of the data discussed in this review was obtained with the generous support of the Italian Association for Cancer Research (AIRC) and of the Italian Ministry of Economy and Finance to the CNR for the Project "FaReBio di Qualità".

Biochem (2007) 71:1811-8. doi: 10.1271/bbb.70062

12. Burch RM, Jelsema C, Axelrod J. Cholera toxin and pertussis toxin stimulate prostaglandin $\mathrm{E}_{2}$ synthesis in a murine macrophage cell line. J Pharmacol Exp Ther (1988) 244:765-73.

13. Gandhi CR, Hanahan DJ, Olson MS. Two distinct pathways of platelet-activating factor-induced hydrolysis of phosphoinositides in primary cultures of rat Kupffer cells. J Biol Chem (1990) 265: 18234-41.

14. Gandhi CR, Stephenson K, Olson MS. A comparative study of endothelin- and platelet-activatingfactor-mediated signal transduction and prostaglandin synthesis in rat Kupffer cells. Biochem J (1992) 281 (Pt 2):485-92.

15. Corda D, Zizza P, Varone A, Filippi BM, Mariggio S. The glycerophosphoinositols: cellular metabolism and biological functions. Cell Mol Life Sci (2009) 66:3449-67. doi:10. 1007/s00018-009-0113-4

16. Patrussi L, Mariggio S, Paccani SR, Capitani N, Zizza P, Corda D, et al., Glycerophosphoinositol-4phosphate enhances SDF-1alphastimulated T-cell chemotaxis through PTK-dependent activation of Vav. Cell Signal (2007) 19:2351-60. doi: 10.1016/j.cellsig.2007.07.014

17. Bonser RW, Siegel MI, McConnell RT, Cuatrecasas P. The appearance of phospholipase and cyclo-oxygenase activities in the human promyelocytic leukemia cell line HL60 during dimethyl sulfoxide-induced differentiation. Biochem Biophys Res Commun (1981) 98:614-20. doi:10.1016/0006291X(81)91158-X

18. Kharbanda S, Nakamura T, Datta $\mathrm{R}$, Sherman ML, Kufe D. Induction of monocytic differentiation by tumor necrosis factor in phorbol ester-resistant KG-1a cells. Cancer Commun (1990) 2:327-32.

19. Falasca M, Marino M, Carvelli A, Iurisci C, Leoni S, Corda D. Changes in the levels of glycerophosphoinositols during differentiation of hepatic and neuronal cells. Eur J Biochem (1996) 241: 386-92. doi:10.1111/j.14321033.1996.00386.x

20. French PJ, Bunce CM, Stephens LR, Lord JM, McConnell FM, Brown $G$, et al., Changes in the levels of inositol lipids and phosphates during the differentiation of HL60 promyelocytic cells towards neutrophils or monocytes. Proc Biol Sci (1991) 245:193-201. doi:10.1098/ rspb.1991.0109

21. Bunce CM, French PJ, Patton WN, Turnell AS, Scott SA, Michell RH, et al., Levels of inositol metabolites within normal myeloid blast cells and changes during their differentiation towards monocytes. Proc Biol Sci (1992) 247:27-33. doi: 10.1098/rspb.1992.0005 
22. Mountford JC, Bunce CM, French PJ, Michell RH, Brown G. Intracellular concentrations of inositol, glycerophosphoinositol and inositol pentakisphosphate increase during hematopoietic cell differentiation. Biochim Biophys Acta (1994) 1222:101-8. doi:10.1016/ 0167-4889(94)90030-2

23. Cantrell DA. GTPases and $\mathrm{T}$ cell activation. Immunol Rev (2003) 192:122-30. doi: 10.1034/j.1600-065X.2003.00028.x

24. Samstag Y, Eibert SM, Klemke M, Wabnitz GH. Actin cytoskeletal dynamics in $\mathrm{T}$ lymphocyte activation and migration. J Leukoc Biol (2003) 73:30-48. doi:10.1189/jlb. 0602272

25. Huang Y, Burkhardt JK. Tcell-receptor-dependent actin regulatory mechanisms. J Cell Sci (2007) 120:723-30. doi: $10.1242 /$ jcs. 000786

26. Mancini R, Piccolo E, Mariggio S, Filippi BM, Iurisci C, Pertile $\mathrm{P}$, et al., Reorganization of actin cytoskeleton by the phosphoinositide metabolite glycerophosphoinositol 4-phosphate. Mol Biol Cell (2003) 14:503-15. doi:10.1091/ mbc.E02-04-0179

27. Filippi BM, Mariggio S, Pulvirenti T, Corda D. SRC-dependent signalling regulates actin ruffle formation induced by glycerophosphoinositol 4-phosphate. Biochim Biophys Acta (2008) 1783: 2311-22. doi:10.1016/j.bbamcr. 2008.07.021

28. Bustelo XR. Vav proteins, adaptors and cell signaling. Oncogene (2001) 20:6372-81. doi:10.1038/sj. onc. 1204780

29. Bokoch GM. Biology of the p21-activated kinases. Annu Rev Biochem (2003) 72:743-81. doi: 10.1146/annurev.biochem.72. 121801.161742

30. Straus DB, Weiss A. Genetic evidence for the involvement of the lck tyrosine kinase in signal transduction through the $\mathrm{T}$ cell antigen receptor. Cell (1992) 70:585-93. doi: 10.1016/0092-8674(92)90428-F

31. Mosenden R, Tasken K. Cyclic AMP-mediated immune regulation - overview of mechanisms of action in $\mathrm{T}$ cells. Cell Signal (2011) 23:1009-16. doi:10.1016/j. cellsig.2010.11.018

32. Iacovelli L, Falasca M, Valitutti S, D'Arcangelo D, Corda D. Glycerophosphoinositol 4-phosphate, a putative endogenous inhibitor of adenylylcyclase. J Biol Chem (1993) 268:20402-7.

33. Falasca M, Carvelli A, Iurisci C, Qiu RG, Symons MH, Corda D. Fast receptor-induced formation of glycerophosphoinositol-4phosphate, a putative novel intracellular messenger in the Ras pathway. Mol Biol Cell (1997) 8:443-53.

34. Howe AK. Regulation of actin-based cell migration by cAMP/PKA. Biochim Biophys Acta (2004) 1692:159-74. doi: 10.1016/j.bbamcr.2004.03.005

35. Dong JM, Leung T, Manser E, Lim L. cAMP-induced morphological changes are counteracted by the activated RhoA small GTPase and the Rho kinase ROKalpha. $J$ Biol Chem (1998) 273:22554-62. doi: 10.1074/jbc.273.35.22554

36. Ellerbroek SM, Wennerberg K, Burridge $K$. Serine phosphorylation negatively regulates RhoA in vivo. J Biol Chem (2003) 278:19023-31. doi:10.1074/jbc.M213066200

37. Salmond RJ, Filby A, Qureshi I, Caserta S, Zamoyska R. T-cell receptor proximal signaling via the Src-family kinases, Lck and Fyn, influences T-cell activation, differentiation, and tolerance. Immunol Rev (2009) 228: 9-22. doi:10.1111/j.1600065X.2008.00745.x

38. Patrussi L, Ulivieri C, Lucherini OM, Paccani SR, Gamberucci A, Lanfrancone L, et al., p52Shc is required for CXCR4-dependent signaling and chemotaxis in T cells. Blood (2007) 110:1730-8. doi:10. 1182/blood-2007-01-068411

39. Patrussi L, Baldari CT. Intracellular mediators of CXCR4-dependent signaling in T cells. Immunol Lett (2008) 115:75-82. doi:10.1016/j. imlet.2007.10.012

40. Finetti F, Savino MT, Baldari CT. Positive and negative regulation of antigen receptor signaling by the Shc family of protein adapters. Immunol Rev (2009) 232:115-34. doi:10.1111/j. 1600-065X.2009.00826.x

41. Kumar A, Humphreys TD, Kremer KN, Bramati PS, Bradfield L, Edgar CE, et al., CXCR4 physically associates with the $\mathrm{T}$ cell receptor to signal in $\mathrm{T}$ cells. Immunity (2006) 25:213-24. doi:10.1016/ j.immuni.2006.06.015

42. Corda D, Zizza P, Varone A Bruzik KS, Mariggio S. The glycerophosphoinositols and their cellular functions. Biochem Soc Trans (2012) 40:101-7. doi: 10.1042/BST20110679

43. Valitutti S, Cucchi P, Colletta G, Di Filippo C, Corda D. Transformation by the k-ras oncogene correlates with increases in phospholipase A2 activity, glycerophosphoinositol production and phosphoinositide synthesis in thyroid cells. Cell Signal (1991) 3:321-32. doi:10.1016/0898 6568(91)90061-X

44. Vicari AP, Caux C. Chemokines in cancer. Cytokine Growth Factor Rev (2002) 13:143-54. doi:10.1016/ S1359-6101(01)00033-8

45. Yu XZ, Levin SD, Madrenas J, Anasetti C. Lck is required for activation-induced $\mathrm{T}$ cell death after TCR ligation with partial agonists. J Immunol (2004) 172:1437-43.

46. Kim MJ, Park MT, Yoon CH, Byun JY, Lee SJ. Activation of Lck is critically required for sphingosineinduced conformational activation of Bak and mitochondrial cell death.
Biochem Biophys Res Commun (2008) 370:353-8. doi:10.1016/j. bbrc.2008.03.084

47. Patrussi L, Giommoni N, Pellegrini M, Gamberucci A, Baldari CT. p66Shc-dependent apoptosis requires Lck and CamKII activity. Apoptosis (2012) 17:174-86. doi:10. 1007/s10495-011-0663-4

48. Shan X, Czar MJ, Bunnell SC, Liu P, Liu Y, Schwartzberg PL, et al. Deficiency of PTEN in Jurkat $\mathrm{T}$ cells causes constitutive localization of Itk to the plasma membrane and hyperresponsiveness to $\mathrm{CD} 3$ stimulation. Mol Cell Biol (2000) 20:6945-57. doi:10.1128/MCB.20. 18.6945-6957.2000

49. Leslie CC. Regulation of arachidonic acid availability for eicosanoid production. Biochem Cell Biol (2004) 82:1-17. doi: 10.1139/o03-080

Conflict of Interest Statement: The authors declare that the research was conducted in the absence of any commercial or financial relationships that could be construed as a potential conflict of interest.

Received: 29 March 2013; paper pending published: 24 April 2013; accepted: 11 July 2013; published online: 29 July 2013. Citation: Patrussi L, Mariggiò S, Corda $D$ and Baldari CT (2013) The glycerophosphoinositols: from lipid metabolites to modulators of T-cell signaling. Front. Immunol. 4:213. doi: 10.3389/fimmu.2013.00213

This article was submitted to Frontiers in $T$ Cell Biology, a specialty of Frontiers in Immunology.

Copyright (c) 2013 Patrussi, Mariggiò, Corda and Baldari. This is an openaccess article distributed under the terms of the Creative Commons Attribution License, which permits use, distribution and reproduction in other forums, provided the original authors and source are credited and subject to any copyright notices concerning any third-party graphics etc. 